\title{
The impact of 18F-FDOPA-PET/MRI image fusion in detecting liver metastasis in patients with neuroendocrine tumors of the gastrointestinal tract
}

\author{
O. Barachini ${ }^{1}$, R. Bernt ${ }^{1}$, S. Mirzaei ${ }^{2}$, C. Pirich ${ }^{3}$, K. Hergan $^{4}$ and S. Zandieh ${ }^{1,4^{*}}$
}

\begin{abstract}
Background: This study assesses the value of image fusion using 18F-fluoro-L-DOPA (18F-DOPA) positron emission tomography (PET) and magnetic resonance imaging (MRI) for examining patients with neuroendocrine tumors (NETs) and a suspicion of metastasis of the liver.

Methods: Eleven patients (five women and six men aged between 20 and 81, with a mean age of 54.6 years) were included in the study. All patients underwent whole-body 18F-DOPA PET examinations and contrast-enhanced MRI with diffusion-weighted sequences (DWS). Image fusion was performed using a semiautomatic voxel-based algorithm. Images obtained using PET and MRI were assessed separately. Side-by-side evaluations of fused PET/MRI images were also performed.

Results: In total, 55 liver lesions (52 liver metastases and 3 benign lesions) were detected in the 11 patients. Sensitivity detection for liver lesions was higher when using PET/CT than when using contrast-enhanced MRI without DWSs and lower than using MRI with DWSs. The sensitivity of PET/MRI image fusion in the detection of liver metastasis was significantly higher than that of MRI with DWSs $(P<0.05)$.

Conclusion: Images of the liver obtained using PET and MRI in patients with NETs exhibited characteristic features. These findings suggest that an appropriate combination of available imaging modalities can optimize patient evaluations.
\end{abstract}

Keywords: Magnetic resonance imaging, Neuroendocrine tumors, Liver, Metastasis, Positron emission tomography

\section{Background}

Neuroendocrine neoplasms (NENs) are a heterogeneous group of tumors that arise in neuroendocrine cells dispersed throughout the body [1]. The main locations of origin for NENs are the gastroenteropancreatic (GEP) system and the lungs. Incidence rates for NENs that develop in the GEP (GEP-NENs) have increased significantly over the last 40 years [2], probably due to increased clinical awareness and improved diagnostic techniques. According to the database of the Surveillance, Epidemiology, and End

\footnotetext{
* Correspondence: zandieh.s@icloud.com

${ }^{1}$ Institute of Radiology and Nuclear Medicine, Hanusch-Hospital,

Heinrich-Collin-Strasse 30, A-1140 Vienna, Austria

${ }^{4}$ Department of Radiology, Paracelsus Medical University of Salzburg,

Salzburg, Austria

Full list of author information is available at the end of the article
}

Results Program, the estimated prevalence of GEP-NENs in the United States is 35 in 100,000 [3]. The Ki-67 protein is a cellular marker for proliferation that is present during all active phases of the cell cycle (G1, S, G2, and mitosis) and absent in resting cells (G0). The Ki-67 index often correlates with the clinical course of an NEN. According to World Health Organization classification, based on their Ki-67 index, NENs are divided into three grades (G1 $\leq 2 \%, G 23-20 \%, G 3>20 \%)$ and G3 NENs are distinguished from neuroendocrine carcinomas (NECs) by the level of histological differentiation, with NETs being well differentiated and NECs poorly differentiated.

Functional NENs associated with hormone excess syndromes, such as insulinomas, glucagonomas, gastrinomas, and vipomas, can be differentiated from nonfunctional 
NENs by the lack of hormonal symptoms; nevertheless, their hormonal production may be detected biochemically [4] - something which is true in the majority of cases. The high density of somatostatin receptors on the surface of NEN cells is a unique feature that is relevant for both diagnostic and therapeutic purposes [4].

Unfortunately, $40-50 \%$ of NEN patients present with distant metastases at the time of initial diagnosis, with the lymph nodes and liver the primary locations of said metastases [5]. The existence of distant metastases is associated with worse prognoses [6], with the extent of the hepatic tumor load being an important prognostic factor that influences therapeutic decisions [6]. Depending on tumor staging and grading, the therapeutic options for GEP-NENs include surgical resection (the only curative therapy) [7], somatostatin analogues, interferon, novel targeted drugs [8], chemotherapy [9], and peptide receptor radionuclide therapy with different radiolabelled somatostatin analogues (e.g., 177Lu-DOTA-octreotate) [10]. Liver metastases can be treated with surgical resection and ablative therapies (e.g., radiofrequency ablation, laser-induced thermotherapy, transarterial embolization, right portal vein embolization, and selective internal radiation therapy) [5].

Therapies related to treating the liver, particularly surgical and ablative methods, necessitate having reliable information about the number, size, location, and extent of liver metastases. Imaging methods that provide accurate information are therefore of the utmost importance. The most commonly used imaging modalities for the evaluation of liver metastases in NENs are contrast-enhanced computed tomography $(\mathrm{CT})$, contrast-enhanced magnetic resonance imaging (MRI) with diffusion-weighted imaging (DWI), and positron emission tomography (PET) in combination with CT (PET/CT) and using various radiotracers.

Contrast-enhanced CT and MRI primarily depict morphology; an additional analysis of contrast enhancement dynamics can provide further insight into the microcirculation of hepatic tissue and metastases [11]. Previous studies have shown that DWI was more sensitive than contrast-enhanced MRI alone and that it identified additional NEN metastases [12, 13]. Depending on the radiotracer used, PET/CT can provide supplementary information about the receptor status and metabolism of the primary tumor and metastases.

The somatostatin analogues 68Ga-DOTATATE, 68GaDOTATOC, and 68Ga-DOTANOC can visualize welldifferentiated NENs with generally low proliferation rates [14], while 18F-fluorodeoxyglucose (18F-FDG) is able to visualize poorly differentiated NENs with high proliferation rates. In clinical settings, however, there is a significant overlap in this regard.

18F-fluoro-L-DOPA (18F-DOPA) PET/CT shows increased DOPA decarboxylase activity in NENs. Since different types of malignant and nonmalignant lesions may show variable expression of somatostatin receptor (SSTR), it may be helpful to use 18F-DOPA as a tracer for catecholamine metabolic pathways when characterizing medullary thyroid cancer, midgut NENs, pheochromocytomas, neuroblastomas, and paragangliomas. The strength of the 18F-fluorodihydroxyphenylalanine (18FFDOPA) radiotracer lies in its ability to detect welldifferentiated and serotonin-secreting tumors in NENs [15-18].

Studies investigating PET/MRI fusion [19] and simultaneous PET/MRI [20-22] using 68Ga-DOTATOC as a tracer have shown promising results in terms of the detection and analysis of GEP-NENs and their metastases. The aim of the present study was to evaluate the practicability and potential of PET/MRI fusion including DWI, using $18 \mathrm{~F}-\mathrm{FDOPA}$ as a radiotracer.

\section{Methods}

Liver MRI examinations were conducted using a 1.5-T machine (Achieva, Philips Medical System, Best, the Netherlands). The MRI sequences included the following: T1-weighted spoiled-gradient echo (SGE) with repetition time $(\mathrm{TR})=150 \mathrm{~ms}$, echo time $(\mathrm{TE})=4.5 \mathrm{~ms}, 90^{\circ}$ flip angle, $6 \mathrm{~mm}$ slice thickness (ST), 20\% gap; and T1-weighted fatsuppressed SGE: TR $=175 \mathrm{~ms}$, TE $=4 \mathrm{~ms}, 90^{\circ}$ flip angle, 8 $\mathrm{mm} \mathrm{ST}, 20 \%$ gap. This began $90-120 \mathrm{~ms}$ after the administration of gadolinium. The following sequences were then run: the T1-weighted out-of-phase SGE $(\mathrm{TR}=135 \mathrm{~ms}, \mathrm{TE}=$ $2.37 \mathrm{~ms}, 90^{\circ}$ flip angle), the T2-weighted fat-suppressed turbo spin-echo $(\mathrm{TR}=1000 \mathrm{~ms}, \mathrm{TE}=69 \mathrm{~ms}, 6 \mathrm{~mm} \mathrm{ST})$, and the T2-weighted fat-suppressed spin-echo $(\mathrm{TR}=$ infinite, $\mathrm{TE}=90 \mathrm{~ms}, 18$ sections acquired in $30 \mathrm{~s}$ of quiet breathing). A phased-array torso coil was used for the examination. A gadoterate meglumine contrast medium (Dotarem, Guerbet, USA) was administered in the form of a rapid bolus injection of $0.1 \mathrm{mmol} / \mathrm{kg}$, followed by a normal saline flush. SGE image acquisition began immediately ( $45 \mathrm{~s}$ after the flush). Dynamic contrast material-enhanced imaging was performed at $20 \mathrm{~s}$ (arterial phase), 60 s (portal venous phase), and $120 \mathrm{~s}$ (equilibrium phase) after injecting gadoterate meglumine.

The parameters for DWI were as follows: TR/TE, 2000/63; b = $600 \mathrm{~s} / \mathrm{mm}^{2}$; FOV, $192 \mathrm{~mm}$; matrix $128 \times$ 128 pixels; ST, $5 \mathrm{~mm}$; intersection gap of $1 \mathrm{~mm}$, with one signal acquired. The acquisition lasted approximately $40 \mathrm{~min}$.

The study group consisted of 11 patients (five women and six men aged between 20 and 81, with a mean age of 54.6 years) (Table 1). All patients were examined with both MRI and 18F-DOPA PET/CT. For the PET examination, the patients fasted for a minimum of $4 \mathrm{~h}$ beforehand, but remained well hydrated orally, and $4 \mathrm{MBq}$ of $18 \mathrm{~F}-\mathrm{FDOPA}$ per kilogram of bodyweight was administered intravenously. After an accumulation time of approximately $30 \mathrm{~min}$, 
Table 1 Characteristics of patients population including primary tumor, WHO grade and prior therapy

\begin{tabular}{lll}
\hline Primary tumor & WHO Grade & Therapy \\
\hline Sigmoid colon & G2 & no prior therapy \\
Bauhin valve & G2 & no prior therapy \\
lleum & G1 & no prior therapy \\
lleum & G1 & no prior therapy \\
lleum & G2 & no prior therapy \\
Colon & G2 & no prior therapy \\
lleum & G2 & no prior therapy \\
lleum & G1 & no prior therapy \\
Colon & G3 & no prior therapy \\
lleum & G2 & no prior therapy \\
lleum & G1 & no prior therapy \\
\hline
\end{tabular}

during which time the patient stayed in a sitting position, an emission scan from the base of the skull to the upper legs was performed.

A Philips Ingenuity TF (Philips Healthcare, PC Best, The Netherlands) PET/CT scanner with an axial field of view of $60 \mathrm{~cm}$ was used. Body scans were obtained, with each table position requiring a scanning time of $1.25 \mathrm{~min}$. The images were reconstructed using a three-dimensional ordered-subsets iterative time-of-flight algorithm, after correction for scatter and attenuation. For the transmission scan $(50 \mathrm{~mA}, 120 \mathrm{kV})$ prior to the emission scan, a collimation of $64 \times 0.625 \mathrm{~mm}$, an ST of $3 \mathrm{~mm}$, and a reconstruction increment of $1.5 \mathrm{~mm}$ were used.

Whole-body acquisition lasted approximately $60 \mathrm{~min}$ for each patient. A fully automatic, multimodal image registration algorithm was used to fuse the images. For both modalities, landmarks used for image fusion included both the liver and the spleen. All the images were adjudged on a Philips workstation using commercial Philips IntelliSpace Portal software. The interval between the MRI and PET examinations was a maximum of 6 weeks.

Image analyses were conducted retrospectively by two experienced radiologists and two experienced nuclear medicine physicians, each of whom had more than 10 years' experience. Kappa statistics were used to evaluate interobserver agreement. Kappa values of $<0.4$, between 0.4 and 0.75 , and $>0.75$ were considered to represent poor, fair-to-good, and excellent agreement, respectively, based on the Fleiss classification [23].

On the MRI scans, metastasis was defined according to previously published criteria. On contrast-enhanced 18F-FDOPA PET/CT, metastases were identified either as: 18F-FDOPA-avid, when they showed higher 18FFDOPA uptake than the normal adjacent liver and when they were seen on contrast-enhanced CT; or as $18 \mathrm{~F}$ FDOPA-non-avid when they were not seen on PET images but appeared on an IV contrast-enhanced CT [24].
Benign liver lesions were defined according to previously published characteristic parameters for MRI and CT results, respectively [25, 26]. A follow-up MRI was performed between 3 and 6 months later.

Biopsies was performed on one lesion per patient, and patients with multiple lesions exhibiting similar characteristics on imaging were presumed to have multifocal metastases of the same histologic type as that of the biopsy. The lesions were also verified in follow-up studies.

\section{Statistical analysis}

Data analysis was performed using IBM SPSS for Windows Version 20 (IBM Corp., Armonk, NY, USA). Patient-based and lesion-based data analyses were performed. For quantitative analyses, ANOVA tests were performed to assess differences between the groups. $P$ values $<0.05$ were considered statistically significant.

\section{Results}

The study found 55 liver lesions in 11 patients, with a mean of $6 \pm 5$ lesions per patient. Of the 11 patients, three exhibited more than 10 liver lesions. Of the 55 lesions, three $(5.5 \%)$ were benign and had an average size of $10 \pm 10 \mathrm{~mm}$. In addition, $52(94.5 \%)$ of the lesions were metastases and had a mean transaxial diameter of $14 \mathrm{~mm}$ (range: $4-41 \mathrm{~mm}$; SD: $11.7 \mathrm{~mm}$ ). Of these 52 liver metastases, 37 (71.1\%) showed pathological 18F-DOPA uptake (Table 2).

The positive predictive value (PPV) for 18F-FDOPA was $93 \%$, while the negative predictive value (NPV) was $12 \%$. The sensitivity for $18 \mathrm{~F}-\mathrm{DOPA}$ was $71 \%$ and the specificity was $40 \%$. Dynamic contrast-enhanced magnetic resonance images (DCE-MRIs) showed that 29 (55.7\%) of the liver metastases were positive, with only two of them in the arterial phase. In the DWI sequence, $46(88.4 \%)$ of the liver metastases were positive. In total, 26 (50\%) of the metastases were hyperintense on the T2-weighted sequence and hypointense on the T1-weighted sequence. Finally, 21 (40.3\%) of the liver metastases were isointense on the T2weighted sequence. The PPV for the DCE-MRI was 91\% and the NPV was $88 \%$. The sensitivity for the DCE-MRI was $91 \%$ and the specificity was $88 \%$.

All of the metastases that remained undetected after using 18F-DOPA PET exhibited no elevated 18F-DOPA uptake and were less than $8 \mathrm{~mm}$ in size $(8 \pm 1 \mathrm{~mm})$, as compared to metastases that were detected using $18 \mathrm{~F}$ DOPA PET, which were $20 \pm 12 \mathrm{~mm}(P<0.01)$. The maximum mean standardized uptake value (SUV) of all lesions was 6.6 (range: 0.9-18.7; SD: 3.9).

Sensitivity was higher for PET/CT than for contrastenhanced MRI without DWI; it was lower for contrastenhanced MRI with DWI. Using DWS for both readers, the sensitivity of PET/MRI image fusion in detecting liver metastases was significantly higher than that of 
Table 2 Characteristics of the liver lesions in patients with NET

\begin{tabular}{|c|c|c|c|c|c|c|c|c|}
\hline $\begin{array}{l}\text { Number } \\
\text { of lesions }\end{array}$ & $\begin{array}{l}\text { Mean } \\
\text { Diameter }\end{array}$ & T2-Weighted & T1-Weighted & Dynamic-Sequence & DWI-Sequence & 18F-DOPA-Uptake & Mean SUV & Diagnosis \\
\hline 52 & $14 \mathrm{~mm}$ & $\begin{array}{l}\text { hyperintense } \\
26(50 \%), \\
\text { hypointense } \\
21(50 \%)\end{array}$ & $\begin{array}{l}\text { hypointense } \\
52(100 \%)\end{array}$ & $\begin{array}{l}\text { positiv } \\
29(55.7 \%)\end{array}$ & $\begin{array}{l}\text { positiv } \\
46(88.4 \%)\end{array}$ & $\begin{array}{l}\text { positiv } \\
37(71.1 \%)\end{array}$ & 6.6 & metastasis \\
\hline 3 & $10 \mathrm{~mm}$ & hyperintense & hypointense & positiv & negativ & positiv & 2.2 & heamangioma \\
\hline
\end{tabular}

PET/CT and MRI $(P<0.05$; Figs. 1 and 2$)$. For PET/ MRI image fusion, the sensitivity was $96 \%$ and the specificity was $88 \%$. The PPV for PET/MRI image fusion was $95 \%$ and the NPV was $88 \%$. Interobserver agreement between the two readers was excellent (kappa value $=0.80$ ).

\section{Discussion}

Our study shows that an appropriate combination of available imaging modalities can optimize patient evaluations. The sensitivity of the PET/MRI image fusion demonstrates that liver metastasis detection is significantly higher when using that technique as opposed to MRI with DWS. The 18F-FDOPA PET tracer provides additional metabolic and functional information that gives valuable additional diagnostic data on patients with NETs [16].

Corrias et al. reported imaging features of malignant abdominal neuroendocrine tumors with rare presentation in four patients [27]. The location and type of NENs could influence the sensitivity of the diagnostic methods. 18FDOPA PET/CT has very high sensitivity to midgut NENs but low sensitivity for foregut NENs. One explanation for the high specificity of 18F-DOPA PET/CT in patients with NENs may be that only neuroendocrine cells can take up, decarboxylate, and store amino acids and their amines. 18F-DOPA is not taken up in significant proportions by inflammatory cells, which is an important advantage over other PET tracers, such as gallium-68-somatostatin analogues and fluorine-18-fluorodeoxyglucose.

Infectious or inflammatory processes may also be incidentally detected on PET/CT examination. There is no reliable SUV threshold that can routinely differentiate infection or inflammation related to malignancy from infectious/inflammatory processes that are hypermetabolic or other non-FDG avid malignancies, such as cystic or necrotic neoplasms [28].

Besides 18F-DOPA PET/CT, the most widely used PET technique for NEN imaging is SSTR PET/CT using gallium-68-somatostatin analogues (i.e., DOTA-NOC, DOTA-TOC, or DOTA-TATE). Recent meta-analysis shows high diagnostic accuracy in this setting $[29,30]$. The use of F-18-FDG-PET/CT is usually limited to patients with high G2 or G3 tumors. Some centers perform PET/CT with both 68Ga-DOTA-SSA and FDG in all patients that have G2 or G3 tumors, because of the prognostic information that is provided when tracking FDG positivity or negativity in tumors.

The different functional characteristics of neuroendocrine cells could also be depicted using 18F-DOPA and gallium-68-somatostatin analogues. The selection for well-differentiated NENs should be guided by the biology of the NENs. Features specific to NENs, such as taking up and decarboxylating l-DOPA and transforming it into dopamine, make 18F-DOPA suitable for depicting NENs with elevated serotonin levels [31, 32].

The receptor-based uptake mechanism in gallium-68somatostatin analogues allows NEN lesions to be depicted independently of their functional activity. Patients could be selected prior to peptide receptor radionuclide therapy by using gallium-68-somatostatin analogues.

The overall superiority of SSR PET/CT compared to 18F-DOPA is evidenced in the few available studies that compare SSR and 18F-DOPA PET/CT in patients with

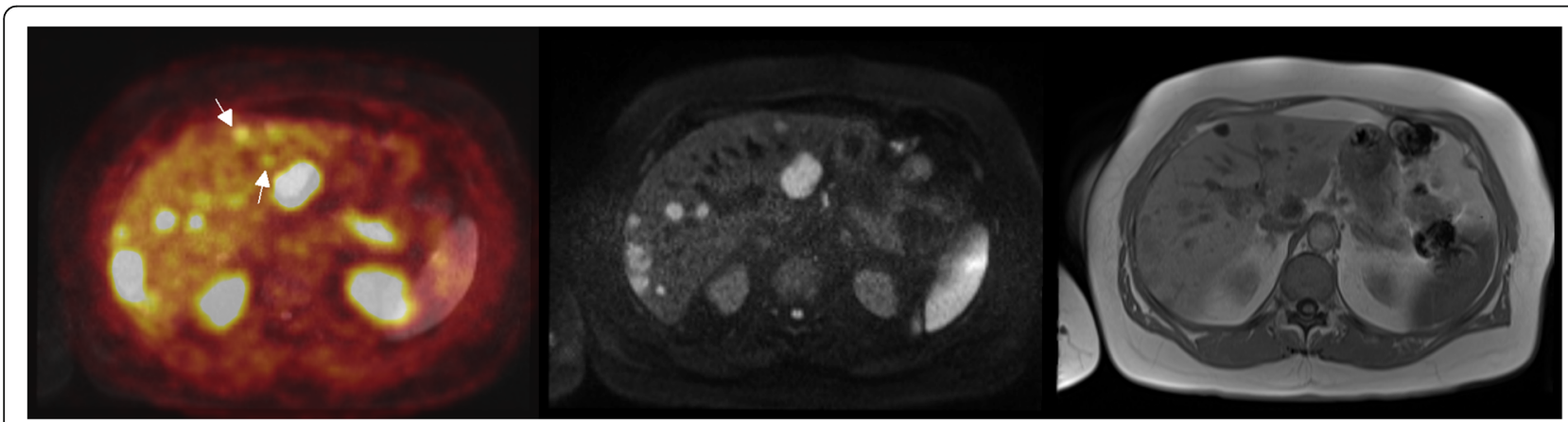

Fig. 1 18F-FDOPA PET/MRI of a patient with primary neuroendocrine tumors shows multiple focal uptakes, suggestive of NET liver metastasis. PET and MRI mismatch was seen on the images 

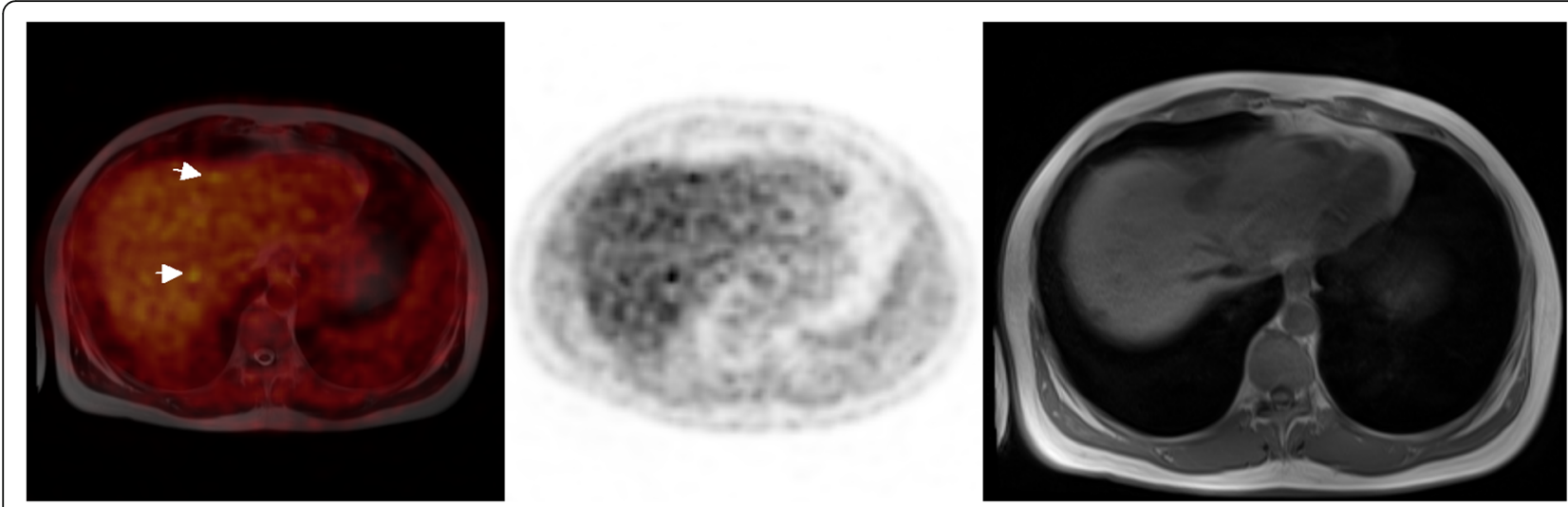

Fig. 2 18F-FDOPA PET/MRI on the patient shows focal uptakes, suggestive of liver metastasis. The MRI/PET mismatch is depicted in this patient

GEP and thoracic NENs. Future comparison studies taking into account the different locations of GEP and thoracic NENs will be necessary to confirm the superiority of SSR PET/CT over 18F-DOPA.

To our knowledge, there have been no significant data comparing SSR and 18F-DOPA PET/CT in patients with Pheochromocytoma and paraganglioma. A study comparing SSR and 18F-DOPA PET/CT in patients with recurrent medullary thyroid cancer illustrated the superiority of 18F-DOPA over SSR PET/CT. More comparison studies are required to establish guidelines for the choice of PET radiopharmaceuticals for evaluating NENs in clinical practice.

Moryoussef et al. conducted a retrospective analysis of 25 abnormal livers and 22 abnormal whole-body standard MRIs [12]. They reported that the addition of DWI sequences to standard liver MRI sequences yielded additional findings in $45 \%$ of patients, with the detection of 1.78 times more new lesions. The same study showed that this resulted in a management change for $18 \%$ of the patients.

Armbruster et al. conducted a DCE-MRI and PET/CT study of 32 patients with NENs and hepatic metastases, using either 18F-FDG or 68Ga-DOTATATE as tracers [33]. They demonstrated that both PET/CT parameters and dynamic contrast-enhanced (DCE)-MRI perfusion parameters showed high diagnostic accuracy in distinguishing between liver metastases and liver tissue. They suggested that the two modalities provided complementary information.

DCE-MRI is currently the state-of-the-art imaging method used for the detection and characterization of liver lesions. High b-values ( $\geq$ b100) produced an increased contrast between the background liver and lesions and supported the detection of focal liver lesions [34]. While DWI alone is less sensitive than a gadoxetic acid-enhanced MRI in detecting liver metastases, it increases the sensitivity of detection for liver metastases to 90.6-95.5\%, when combined with DCE-MRI [35].
Contrast-enhanced MDCT features correlate with histological findings and enable differentiation between G1 and G2 pNETs during preoperative examinations [36]. Third generation DECT, with an assessment of iodine uptake, improves the differentiation of hepatic NET metastasis and hepatocellular carcinoma (HCC) in noncirrhotic livers, as shown in a study by Kaltenbach et al. that examined 46 patients with either hepatic NEN metastasis or HCC, all of whom had undergone liver DECT in a retrospective study [37].

In the present study, we investigated the value of image fusion with 18F-DOPA and MRI in patients with NETs and a suspicion of metastasis of the liver. The results were significant when comparing the detection rate of liver metastasis using PET/MRI image fusion and PET/CT or MRI with DWSs $(P<0.05)$. Gaspard et al. reported that DW MRI was more sensitive than fat-suppressed T2weighted fast spin echo (FSE) and gadolinium-enhanced T1-weighted imaging, and that a combination of sequences improved the detection of liver metastases [13]. In their study, the best results were obtained by combining DW, fat-suppressed T2-weighted FSE, and gadolinium-enhanced T1-weighted sequences, and they detected a total of 162 liver metastases in 41 patients. The findings of the present study were in accordance with the results of the study by Gaspard et al. A novel aspect of the present study was the use of additional information provided by 18F-FDOPA PET, something that has not been reported previously.

The use of diagnostic imaging continues to expand and is routinely employed in clinical settings. The present study demonstrates the potential role that $18 \mathrm{~F}$ FDOPA PET/MRI fusion could play in detecting liver metastasis in patients with NETs of the gastrointestinal tract. A limitation of the present study was the delay (a maximum of 6 weeks) between the MRI and PET examinations. This delay may have had a partial response effect on the metabolic activity. However, as shown in Table 1, there was still enhanced uptake, indicating 
disease activity in these areas. Our data show that 18FFDOPA PET/MRI fusion imaging may be useful in the diagnosis of liver metastasis in patients, however, the small patient population and the retrospective nature of the study do not allow us to draw any detailed conclusions. Larger studies with long-term follow-up are needed to confirm the effectiveness of the hybrid approach.

Overall, the findings of the present study confirm that 18F-FDOPA PET-MRI is clinically useful for the detection of NET liver metastasis.

\section{Conclusion}

The fusion of 18F-FDOPA-PET and MRI allows clinicians to obtain a morphofunctional map in patients with NET. The data show that 18F-FDOPA PET/MRI fusion imaging may be useful in the diagnosis of liver metastasis in patients with NETs of the gastrointestinal tract.

\section{Abbreviations}

18 F-FDG-PET: 18 F-fluor-desoxyglucose positron emission tomography; CT: Computed tomography; DWSs: Diffusion-weighted sequences; MRI: Magnetic resonance imaging; NETs: Neuroendocrine tumors

\section{Acknowledgements}

Not applicable.

\section{Authors' contributions}

$\mathrm{OB}$ and $\mathrm{SZ}$ conceived the study and design. SZ, OB, and RB undertook the acquisition of data. $\mathrm{SZ}$ and $\mathrm{OB}$ analyzed and interpreted the data and drafted the manuscript. SM, CP, and $\mathrm{KH}$ performed critical revision of the manuscript. SZ and KH supervised the study. All the authors read and approved the final manuscript.

\section{Funding}

The study was not funded.

\section{Availability of data and materials}

Data to replicate findings are presented in the tables of the main paper. Due to patient privacy protection, any additional materials from the study are available only upon individual request directed to the corresponding author.

\section{Ethics approval and consent to participate}

The local ethics committee of Vienna (Magistrat der Stadt Wien Magistratsabteilung 15) granted ethical approval for this study. Informed written consent was obtained from all the participants. This study conformed to the Declaration of Helsinki.

\section{Consent for publication}

Not applicable.

\section{Competing interests}

The authors declare that they have no competing interests.

\section{Author details}

'Institute of Radiology and Nuclear Medicine, Hanusch-Hospital, Heinrich-Collin-Strasse 30, A-1140 Vienna, Austria. ${ }^{2}$ Department of Nuclear Medicine with PET-Center, Wilhelminen-Hospital, Vienna, Austria. ${ }^{3}$ Department of Nuclear Medicine, Paracelsus Medical University of Salzburg, Salzburg, Austria. ${ }^{4}$ Department of Radiology, Paracelsus Medical University of Salzburg, Salzburg, Austria.
Received: 22 August 2019 Accepted: 11 February 2020

Published online: 24 February 2020

\section{References}

1. llett $\mathrm{EE}$, et al. Neuroendocrine carcinomas of the gastroenteropancreatic system: a comprehensive review. Diagnostics (Basel, Switzerland). 2015;5: 119-76.

2. Fraenkel M, Kim M, Faggiano A, de Herder WW, Valk GD. Incidence of gastroenteropancreatic neuroendocrine tumours: a systematic review of the literature. Endocr Relat Cancer. 2014;21:153-63.

3. Yao JC, et al. One hundred years after "carcinoid": epidemiology of and prognostic factors for neuroendocrine tumors in 35,825 cases in the United States. J Clin Oncol. 2008;26:3063-72.

4. Anlauf M. Neuroendocrine neoplasms of the gastroenteropancreatic system: pathology and classification. Horm Metab Res. 2011;43:825-31.

5. Pavel $\mathrm{M}$, et al. ENETS consensus guidelines update for the management of distant metastatic disease of intestinal, pancreatic, bronchial neuroendocrine neoplasms (NEN) and NEN of unknown primary site. Neuroendocrinology. 2016;103:172-85.

6. Auernhammer CJ, Goke B. Therapeutic strategies for advanced neuroendocrine carcinomas of jejunum/ileum and pancreatic origin. Gut. 2011:60:1009-21.

7. Partelli S, et al. ENETS consensus guidelines for standard of care in neuroendocrine tumours: surgery for small intestinal and pancreatic neuroendocrine tumours. Neuroendocrinology. 2017;7.105(3):255-265. https://doi.org/10.1159/000464292.

8. Pavel $\mathrm{M}$, et al. ENETS consensus guidelines for the standards of care in neuroendocrine neoplasms: systemic therapy - biotherapy and novel targeted agents. Neuroendocrinology. 2017;105(3):266-280. https://doi.org/ $10.1159 / 000471880$

9. Garcia-Carbonero R, et al. ENETS consensus guidelines for the standards of care in neuroendocrine neoplasms. Systemic therapy 2: chemotherapy. Neuroendocrinology. 2017;105(3):281-294. https://doi.org/10.1159/ 000473892.

10. Hicks RJ, et al. ENETS consensus guidelines for the standards of care in neuroendocrine neoplasia: peptide receptor radionuclide therapy with radiolabeled somatostatin analogues. Neuroendocrinology. 2017;105(3):295309. https://doi.org/10.1159/000475526.

11. Armbruster M, et al. Evaluation of neuroendocrine liver metastases: a comparison of dynamic contrast-enhanced magnetic resonance imaging and positron emission tomography/computed tomography. Investig Radiol. 2014;49:7-14

12. Moryoussef F, et al. Impact of liver and whole-body diffusion-weighted MRI for neuroendocrine tumors on patient management: a pilot study. Neuroendocrinology. 2017;104:264-72.

13. d'Assignies $\mathrm{G}$, et al. High sensitivity of diffusion-weighted MR imaging for the detection of liver metastases from neuroendocrine tumors: comparison with T2-weighted and dynamic gadolinium-enhanced MR imaging. Radiology. 2013;268:390-9.

14. Rufini $V$, et al. Role of PET/CT in the functional imaging of endocrine pancreatic tumors. Abdom Imaging. 2012;37:1004-20.

15. Fiebrich $H B$, de Jong JR, Kema IP, et al. Total 18F-dopa PET tumour uptake reflects metabolic endocrine tumour activity in patients with a carcinoid tumour. Eur J Nucl Med Mol Imaging. 2011;38:1854-61.

16. Imperiale A, et al. 18F-fluorodihydroxyphenylalanine PET/CT in patients with neuroendocrine tumors of unknown origin: relation to tumor origin and differentiation. J Nucl Med. 2014;55:367-72.

17. Koopmans KP, et al. Staging of carcinoid tumours with 18F-DOPA PET: a prospective, diagnostic accuracy study. Lancet Oncol. 2006;7:728-34.

18. Koopmans KP, et al. Improved staging of patients with carcinoid and islet cell tumors with 18F-dihydroxy-phenyl-alanine and 11C-5-hydroxytryptophan positron emission tomography. J Clin Oncol. 2008;26:1489-95.

19. Schreiter NF, et al. Evaluation of the potential of PET-MRI fusion for detection of liver metastases in patients with neuroendocrine tumours. Eur Radiol. 2012:22:458-67.

20. Beiderwellen KJ, et al. Simultaneous 68Ga-DOTATOC PET/MRI in patients with gastroenteropancreatic neuroendocrine tumors: initial results. Investig Radiol. 2013;48:273-9.

21. Gaertner FC, et al. Evaluation of feasibility and image quality of 68GaDOTATOC positron emission tomography/magnetic resonance in 
comparison with positron emission tomography/computed tomography in patients with neuroendocrine tumors. Investig Radiol. 2013;48:263-72.

22. Hope TA, et al. Simultaneous (68)Ga-DOTA-TOC PET/MRI with gadoxetate disodium in patients with neuroendocrine tumor. Abdom Imaging. 2015;40: 1432-40.

23. Fleiss JL, Levin B, Paik MC. Statistical methods for rates and proportions. 3rd ed. Hoboken: Wiley; 2003.

24. Yu MH, Lee JM, Hur BY, et al. Gadoxetic acid-enhanced MRI and diffusionweighted imaging for the detection of colorectal liver metastases after neoadjuvant chemotherapy. Eur Radiol. 2015;25:2428-36.

25. Grazioli L, Ambrosini R, Frittoli B, et al. Primary benign liver lesions. Eur 」 Radiol. 2017;95:378-98.

26. Ronot M, Clift AK, Baum RP, Singh A, Kulkarni HR, Frilling A, Vilgrain V. Morphological and functional imaging for detecting and assessing the resectability of neuroendocrine liver metastases. Neuroendocrinology. 2018; 106:74-88. https://doi.org/10.1159/000479293.

27. Corrias $\mathrm{G}$, Monti S, Horvat N, et al. Imaging features of malignant abdominal neuroendocrine tumors with rare presentation. Clin Imaging. 2018;51:59-64.

28. Kinahan PE, Fletcher JW. Positron emission tomography-computed tomography standardized uptake values in clinical practice and assessing response to therapy. Semin Ultrasound CT MR. 2010;31(6):496-505.

29. Carideo L, Prosperi D, Panzuto F, et al. Role of combined [68Ga]Ga-DOTASST analogues and [18F] FDG PET/CT in the management of GEP-NENs: a systematic review. J Clin Med. 2019:8(7):1032.

30. Tirosh A, Kebebew E. The utility of 68Ga-DOTATATE positron-emission tomography/computed tomography in the diagnosis, management, follow-up and prognosis of neuroendocrine tumors. Future Oncol. 2018;14(2):111-22.

31. Beuthien-Baumann B. 18F-DOPA-PET in neuroendocrine tumours (NET). Nuklearmediziner. 2009;32(2):131-4.

32. Hoegerle S, et al. Whole-body $18 \mathrm{~F}$ dopa PET for detection of gastrointestinal carcinoid tumors. Radiology. 2001;220(2):373-80.

33. Armbruster M, Zech CJ, Sourbron S, Ceelen F, Auernhammer CJ, Rist C, Haug A, Singnurkar A, Reiser MF, Sommer WH. Diagnostic accuracy of dynamic gadoxetic-acid-enhanced MRI and PET/CT compared in patients with liver metastases from neuroendocrine neoplasms. J Magn Reson Imaging. 2014;40(2):457-66

34. Shenoy-Bhangle A, Baliyan V, Kordbacheh H, Guimaraes AR, Kambadakone A. Diffusion weighted magnetic resonance imaging of liver: principles, clinical applications and recent updates. World J Hepatol. 2017:9(26):1081-91.

35. Vilgrain V, Esvan M, Ronot M, Caumont-Prim A, Aubé C, Chatellier G. A meta-analysis of diffusion-weighted and gadoxetic acid-enhanced MR imaging for the detection of liver metastases. Eur Radiol. 2016;26:4595-615.

36. Belousova E, Karmazanovsky G, Kriger A, et al. Contrast-enhanced MDCT in patients with pancreatic neuroendocrine tumours: correlation with histological findings and diagnostic performance in differentiation between tumor grades. Clin Radiol. 2017;72(2):150-8.

37. Kaltenbach $B$, Wichmann JL, Pfeifer $S$, et al. Iodine quantification to distinguish hepatic neuroendocrine tumor metastasis from hepatocellular carcinoma at dual-source dual-energy liver CT. Eur J Radiol. 2018;105:20-4.

\section{Publisher's Note}

Springer Nature remains neutral with regard to jurisdictional claims in published maps and institutional affiliations.

Ready to submit your research? Choose BMC and benefit from:

- fast, convenient online submission

- thorough peer review by experienced researchers in your field

- rapid publication on acceptance

- support for research data, including large and complex data types

- gold Open Access which fosters wider collaboration and increased citations

- maximum visibility for your research: over $100 \mathrm{M}$ website views per year

At BMC, research is always in progress.

Learn more biomedcentral.com/submissions 